\title{
Calculation of primordial abundances of light nuclei including a heavy sterile neutrino
}

\author{
M. E. Mosquera, ${ }^{a, b}$ O. Civitarese $e^{b, 1}$ \\ ${ }^{a}$ Facultad de Ciencias Astronómicas y Geofísicas, Universidad Nacional de La Plata, \\ Paseo del Bosque, (1900) La Plata, Argentina \\ ${ }^{b}$ Department of Physics, University of La Plata, \\ c.c. 67 (1900), La Plata, Argentina \\ E-mail: mmosquera@fcaglp.unlp.edu.ar, osvaldo.civitarese@fisica.unlp.edu.ar
}

\begin{abstract}
We include the coupling of a heavy sterile neutrino with active neutrinos in the calculation of primordial abundances of light-nuclei. We calculate neutrino distribution functions and primordial abundances, as functions depending on a renormalization of the sterile neutrino distribution function $(a)$, the sterile neutrino mass $\left(m_{s}\right)$ and the mixing angle $(\phi)$. Using the observable data, we set constrains on these parameters, which have the values $a<0.60, \sin ^{2} \phi=0.15$ and $m_{s} \approx 4 \mathrm{keV}$, for a fixed value of the baryon to photon ratio. When the baryon to photon ratio is allowed to vary, its extracted value is in agreement with the values constrained by Planck observations and by the Wilkinson Microwave Anisotropy Probe (WMAP). It is found that the anomaly in the abundance of ${ }^{7} \mathrm{Li}$ persists, in spite of the inclusion of a heavy sterile neutrino.
\end{abstract}

\footnotetext{
${ }^{1}$ Corresponding author.
} 


\section{Contents}

1 Introduction $\quad 1$

2 Formalism $r$

3 Results 13

3.1 Results with $\eta_{B}$ fixed $\quad 4$

3.2 Results with $\eta_{B}$ variable 25

\section{Introduction}

The observational data obtained by the WMAP Collaboration [1] and by Planck [2] signalled the existence of a deficit in the abundance of primordial lithium, which cannot be explained in the context of the standard Big Bang Nucleosynthesis (BBN). Several authors have studied the problem from different points of view: i) the turbulent transport in the radiative zones of stars [3]; ii) the existence of a stellar lithium depletion that depends on the mass of the star $[4,5]$; iii) the nuclear physics aspects of the abundance of ${ }^{7} \mathrm{Li}[6-8]$; iv) variation of fundamental constants [9-13], among others, but the question is still open since none of these possible explanations, although plausible, provides a complete solution to the problem.

In previous works, we have analysed the effect of the inclusion of light sterile-neutrinos during the first three minutes of the Universe [14-17], to calculate primordial abundances as a function of the active-sterile mixing parameters in the two-state scheme, the $3+1$ scheme and in the $3+2$ scheme. We have also analysed the case where the sterile-neutrino might have a variable normalization constant in its occupation factor [17]. The results of the calculations indicate that the value of the normalization constant should be of the order of (or smaller than) 0.65 and that the mixing angle must be zero, in order to be consistent with the observational data.

In this work, we extent our previous study [17], by considering a heavy sterile neutrino coupled to the active ones. To perform the calculation we solve the evolution equation of an expanding Universe, including neutrino oscillations in the decay rates. Using the available observational data we set limits on the sterile-neutrino sector.

This work is organized as follows. In Section 2 we present the formalism and in Section 3 we present and discuss the results of primordial abundances as functions of the baryon density, the sterile neutrino occupation factor, and the active-sterile mixing angle. Finally, in Section 4, the conclusions are drawn.

\section{Formalism}

The matrix which relates neutrino mass-eigenstates and neutrino flavour-eigenstates is the unitary matrix [18]

$$
U=\left(\begin{array}{cccc}
c_{12} c_{13} \cos \phi & c_{13} s_{12} \cos \phi & s_{13} \cos \phi & \sin \phi \\
\alpha & \delta & s_{23} c_{13} & 0 \\
\epsilon & \lambda & c_{23} c_{13} & 0 \\
-c_{13} c_{12} \sin \phi & -c_{13} s_{12} \sin \phi & -s_{13} \sin \phi & \cos \phi
\end{array}\right)
$$


where $i, j=1,2,3$ denote mass eigenstates, $s_{i j}\left(c_{i j}\right)$ stands for $\sin \theta_{i}\left(\cos \theta_{i j}\right), \alpha=-s_{12} c_{23}-$ $s_{13} c_{12} s_{23}, \delta=c_{23} c_{12}-s_{13} s_{12} s_{23}, \epsilon=s_{23} s_{12}-s_{13} c_{12} c_{23}, \lambda=-s_{23} c_{12}-s_{13} s_{12} c_{23}$, and $\phi$ is the mixing-angle of the lowest mass-eigenstate with the sterile neutrino. The inclusion of a sterile-neutrino affects the statistical occupation factor of active neutrinos, quantities which are crucial for the determination of primordial abundances. In order to compute these new factors, one must solve the equation [19]

$$
\left(\frac{\partial f}{\partial t}-\mathrm{H} p \frac{\partial f}{\partial p}\right)=-\imath\left[\mathcal{H}_{0}, f\right]
$$

where $f$ is the $4 \times 4$ matrix of the occupation factors, $t$ is the time, $\mathrm{H}$ is the expansion rate of the Universe $\left(\mathrm{H}=\mu_{P} \mathrm{~T}^{2}\right)$, and $\mathcal{H}_{0}$ is the unperturbed mass term of the neutrino Hamiltonian in the rest frame. We have assumed that at the temperature $\mathrm{T}_{0}=5 \mathrm{MeV}$ the occupation factors for all neutrinos in the usual flavour representation (namely electron-neutrino, muonneutrino and tau-neutrino, respectively) are Fermi-Dirac distributions for massless particles with energy $\mathrm{E}_{\nu}=p(c=1$ everywhere). For the sterile neutrino, being massive, we assume that its occupation factor is a Fermi-Dirac distribution with energies $\left(\mathrm{E}_{s}=\sqrt{m_{s}^{2}+p^{2}}\right)$. The relationship between the sterile neutrino mass and the mass of the mass-eigenstates, $m_{i}$, can be written as $m_{s}=\sum_{i} m_{i}\left|U_{4 i}\right|^{2}$. This occupation factor is further renormalized by a constant factor $a[20]$ which varies between 0 and 1 . The initial condition for the occupation factors in the mass-eigenstates representation is written

$$
\begin{aligned}
\left.\left(\begin{array}{llll}
f_{11} & f_{12} & f_{13} & f_{14} \\
f_{21} & f_{22} & f_{23} & f_{24} \\
f_{31} & f_{32} & f_{33} & f_{34} \\
f_{41} & f_{42} & f_{43} & f_{44}
\end{array}\right)\right|_{T_{0}}= & \frac{1}{1+e^{p / T_{0}}}\left(\begin{array}{cccc}
\cos \phi^{2} & 0 & 0 & -\cos \phi \sin \phi \\
0 & 1 & 0 & 0 \\
0 & 0 & 1 & 0 \\
-\cos \phi \sin \phi & 0 & 0 & \sin \phi^{2}
\end{array}\right) \\
& +\frac{a}{1+e^{E_{s} / T_{0}}}\left(\begin{array}{cccc}
\sin \phi^{2} & 0 & 0 & \cos \phi \sin \phi \\
0 & 0 & 0 & 0 \\
0 & 0 & 0 & 0 \\
\cos \phi \sin \phi & 0 & 0 & \cos \phi^{2}
\end{array}\right)
\end{aligned}
$$


The solutions, for flavour-eigenstates, are

$$
\begin{aligned}
f_{e e}= & \frac{1}{1+e^{\frac{p}{T}}}\left\{1+\frac{1}{2} \cos ^{2} \theta_{12} \cos ^{2} \theta_{13} \sin ^{2} 2 \phi \frac{e^{\frac{p}{T}}-\beta_{0}}{1+e^{-\beta_{0}}}[1-\cos \gamma]\right\} \\
& -\frac{1}{2} \cos ^{2} \theta_{12} \cos ^{2} \theta_{13} \sin ^{2} 2 \phi[1-\cos \gamma] \frac{1-a}{1+e^{\beta_{0}}}, \\
f_{\mu \mu}= & \frac{1}{1+e^{\frac{p}{T}}}\left\{1+\frac{1}{2} \sin ^{2} \theta_{12} \cos ^{2} \theta_{13} \sin ^{2} 2 \phi \frac{e^{\frac{p}{T}-\beta_{0}}-1}{1+e^{-\beta_{0}}}[1-\cos \gamma]\right\} \\
& -\frac{1}{2} \sin ^{2} \theta_{12} \cos ^{2} \theta_{13} \sin ^{2} 2 \phi[1-\cos \gamma] \frac{1-a}{1+e^{\beta_{0}}}, \\
f_{\tau \tau}= & \frac{1}{1+e^{\frac{p}{T}}}\left\{1+\frac{1}{2} \sin ^{2} \theta_{13} \sin ^{2} 2 \phi \frac{e^{\frac{p}{T}}-\beta_{0}}{1+e^{-\beta_{0}}}[1-\cos \gamma]\right\} \\
& -\frac{1}{2} \sin ^{2} \theta_{13} \sin ^{2} 2 \phi[1-\cos \gamma] \frac{1-a}{1+e^{\beta_{0}}}, \\
f_{s s}= & \frac{1}{1+e^{\beta_{0}}}\left\{-\frac{1}{2} \sin ^{2} 2 \phi \frac{e^{\beta_{0}-\frac{p}{T}}-1}{1+e^{-\frac{p}{T}}}[1-\cos \gamma]\right\} \\
& +\frac{1}{2} \sin ^{2} 2 \phi \frac{\lambda}{1+e^{\beta_{0}}}[1-\cos \gamma]+\frac{a}{1+e^{\beta_{0}}},
\end{aligned}
$$

where

$$
\begin{aligned}
\gamma=\frac{1}{\mu_{P}} & {\left[-\frac{m_{1}^{2} T}{6 p}\left(\frac{1}{T^{3}}-\frac{1}{T_{0}^{3}}\right)+\frac{p}{T}\left(\frac{1}{T}-\frac{1}{T_{0}}\right)-\frac{1}{2 T^{2}} \sqrt{m_{4}^{2}+p^{2}}+\frac{1}{2 T_{0}^{2}} \sqrt{m_{4}^{2}+\left(\frac{p T_{0}}{T}\right)^{2}}\right.} \\
& \left.-\frac{p^{2}}{2 m_{4} T^{2}} \ln \left(\frac{T_{0}}{T} \frac{m_{4}+\sqrt{m_{4}^{2}+p^{2}}}{m_{4}+\sqrt{m_{4}^{2}+\left(\frac{p T_{0}}{T}\right)^{2}}}\right)\right],
\end{aligned}
$$

and $\beta_{0}=T_{0}^{-1} \sqrt{m_{s}^{2}+\left(\frac{p T_{0}}{T}\right)^{2}}$.

\section{Results}

In order to obtain the primordial abundances as functions of the active-sterile neutrino mixing parameters, we have modified the numerical code of Kawano [21, 22]. The active neutrino mixing parameters were extracted from the SNO, SK, GNO, CHOOZ, DAYA BAY and DOUBLE CHOOZ experiments [23-28]. The light-neutrino mass was fixed at the square root of the lowest squared mass difference. The active-sterile mixing angle was fixed at the value $\sin ^{2} 2 \phi=0.15$. We have considered two different cases; (i) by fixing the baryon density at the value determined from WMAP [1], and (ii) varying $\eta_{B}$.

To obtain the best value for the parameters of the sterile-neutrino sector we have performed a $\chi^{2}$ minimization in the corresponding parametric space. The observational data for deuterium have been extracted from Refs. [29-33]. We use the data from Refs. [34-39] for ${ }^{4} \mathrm{He}$ and, for ${ }^{7} \mathrm{Li}$ we have considered the data given by Refs. [40-43]. Regarding the consistency of the data, we have followed the treatment of Ref. [44] and increased the errors by a fixed factor $\Theta_{4} \mathrm{He}=1.30$, for the other cases the errors were not changed. 


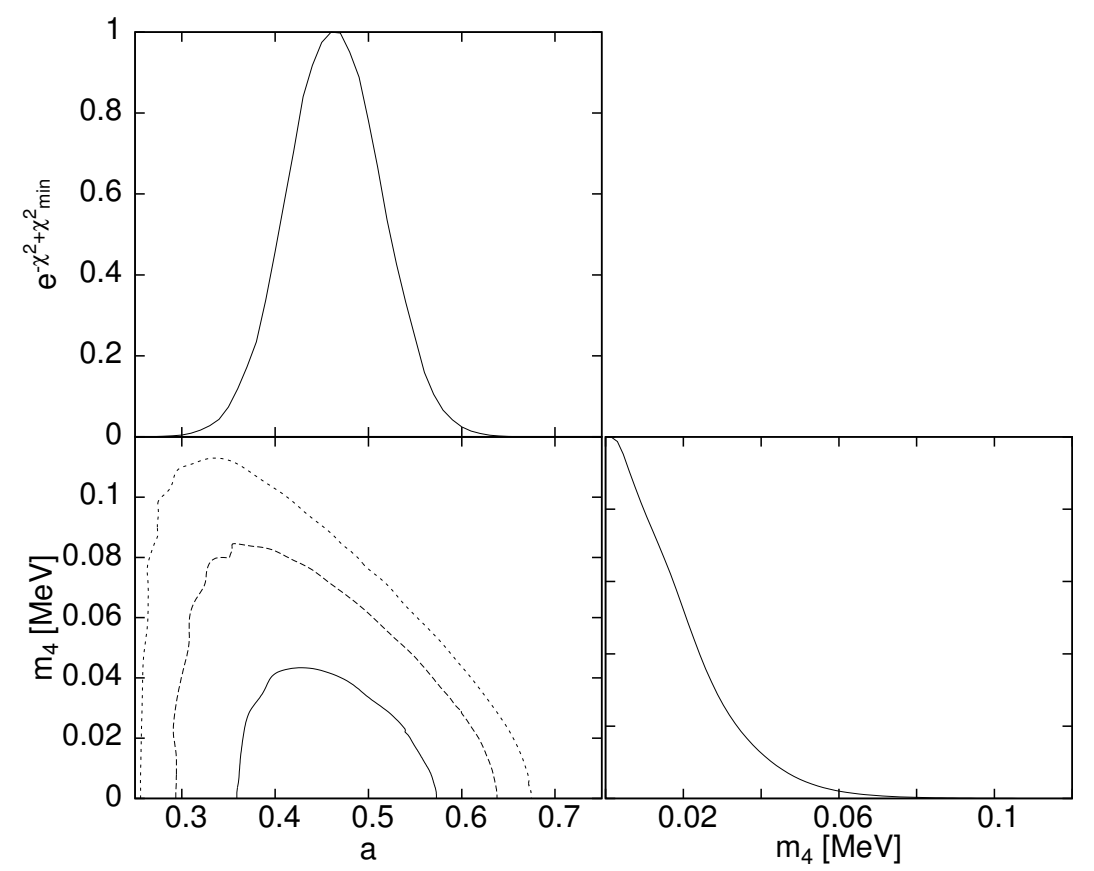

Figure 1. Likelihood and $1 \sigma, 2 \sigma$ and $3 \sigma$ contour plots for the parameter $a$ (renormalization of the sterile neutrino occupation factor) and the sterile-neutrino mass, when all data are considered in the $\chi^{2}$-test. The mixing angle $\phi$ has been fixed at the value $\sin ^{2} 2 \phi=0.15$.

\subsection{Results with $\eta_{B}$ fixed}

In this section we present the results of the calculation of primordial abundances performed as a function of two parameters: the sterile-neutrino mass and the renormalization factor $a$. The value of baryon density was fixed at the WMAP value. We performed a $\chi^{2}$-analysis in order to obtain the best-fit value of the parameters. The results are the following

- All data

$$
\begin{aligned}
a & =0.46_{-0.06}^{+0.08}, \\
m_{4} & =0.003_{-0.003}^{+0.022} \mathrm{MeV}, \\
\chi^{2} /(N-2) & =10.76 .
\end{aligned}
$$

- All data but ${ }^{7} \mathrm{Li}$

$$
\begin{aligned}
a & =0.37 \pm 0.06, \\
m_{4} & =0.004_{-0.004}^{+0.021} \mathrm{MeV}, \\
\chi^{2} /(N-2) & =3.23 .
\end{aligned}
$$

The first set of results have been obtained by taking all data on primordial abundances, and the second one is the set of results obtained by removing the data on the abundance of lithium. Figures 1 and 2 show the contour plots in the parametric-plane $\left(m_{4}, a\right)$ and the likelihood contour plots.

The statistical analysis shows that the data on lithium may not be consistent with the other data on primordial abundances, since $\frac{\chi^{2}(\text { with Li })}{\chi^{2} \text { (without Li) }} \approx 3$. However, both sets yield almost 


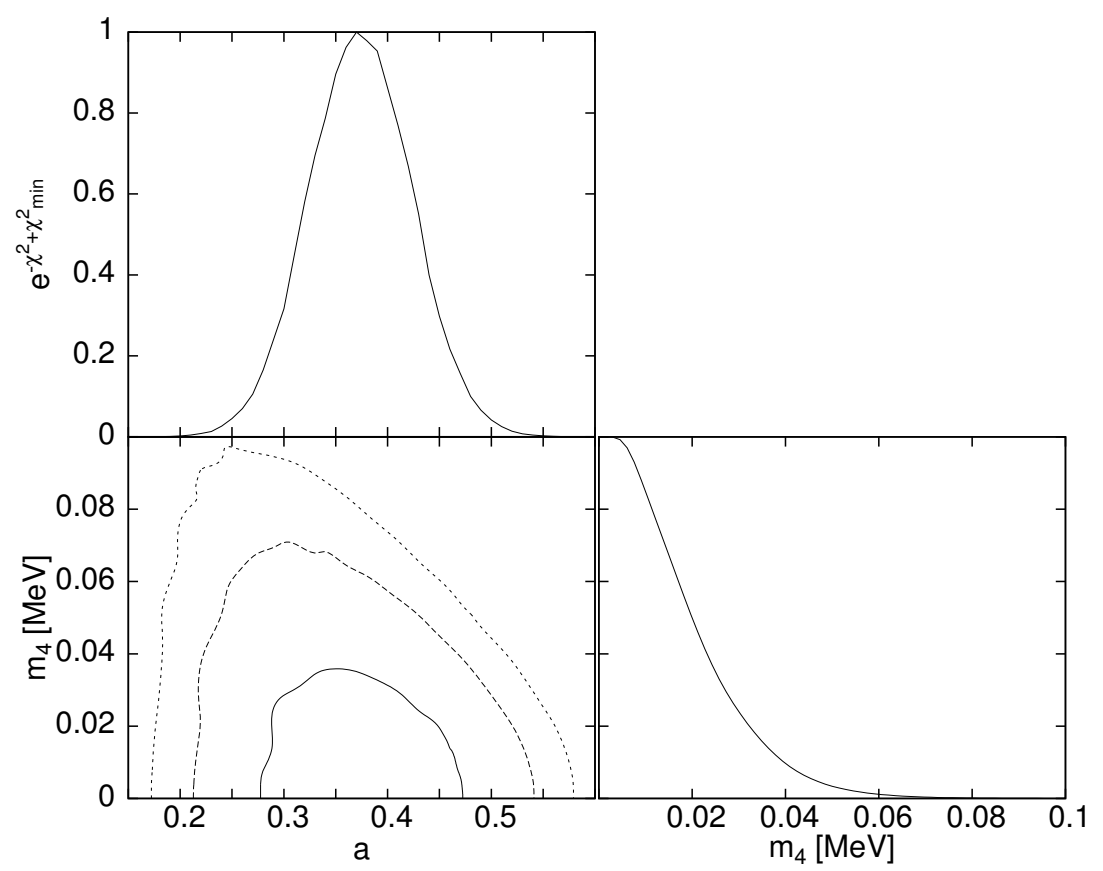

Figure 2. Likelihood and $1 \sigma, 2 \sigma$ and $3 \sigma$ contour plots, in the same notation of Figure 1 , when the data of ${ }^{7} \mathrm{Li}$ are not considered in the $\chi^{2}$-test. The mixing angle $\phi$ has been fixed at the value $\sin ^{2} 2 \phi=0.15$.

the same value for the sterile-neutrino mass and comparable values for the renormalization factor of the sterile-neutrino occupation.

\subsection{Results with $\eta_{B}$ variable}

The results obtained by allowing the variation of the baryon to photon ratio, $\eta_{B}$, are the following:

$$
\begin{aligned}
\eta_{B} & =\left(6.20_{-0.07}^{+0.06}\right) \times 10^{-10}, \\
a & =0.53 \pm 0.09, \\
m_{4} & =0.000^{+0.040} \mathrm{MeV}, \\
\chi^{2} /(N-3) & =11.40 .
\end{aligned}
$$

Figure 3 shows the contour plots resulting from the calculations. The extracted value for $\eta_{B}$ is in good agreement with data, the renormalization parameter $a$ is somehow larger than the one obtained with a fixed value of $\eta_{B}[1,2]$, but the fit favors a massless sterile-neutrino. The $\chi^{2}$-value for this case is similar to the one corresponding to the minimization with a fix $\eta_{B}$ when all primordial species are considered. Since we have three parameters to adjust and three set of data, we cannot, for the case of variable baryon to photon ratio, excluded the data on lithium.

\section{Conclusion}

The presence of an extra heavy-neutrino affects the primordial abundances produced during the first three minutes of the Universe. We have calculated the occupation factors for active 


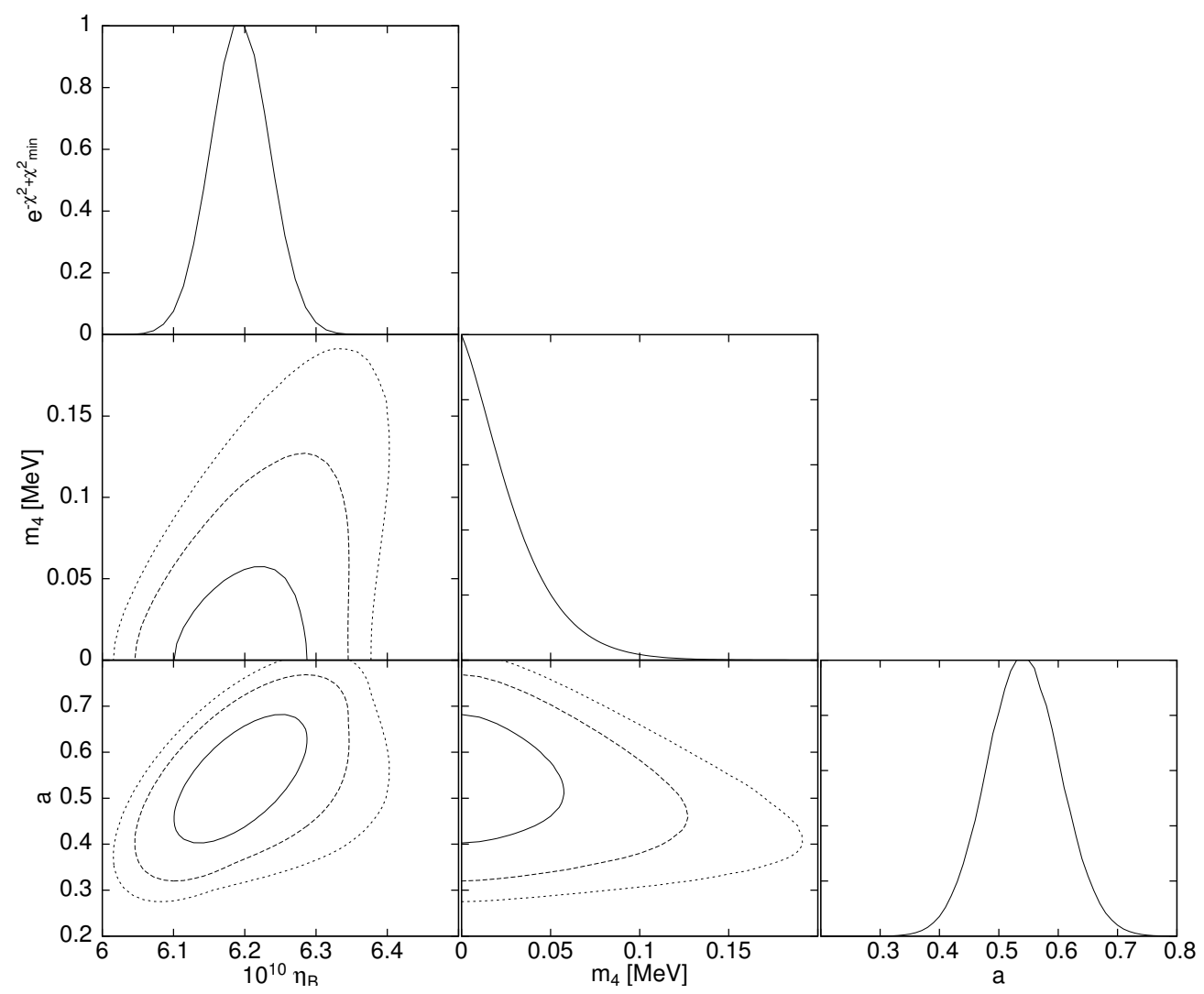

Figure 3. Likelihood and $1 \sigma, 2 \sigma$ and $3 \sigma$ contour plots for $\eta_{B}, m_{4}$ and the parameter $a$. The mixing angle $\phi$ has been fixed at the value $\sin ^{2} 2 \phi=0.15$.

and sterile-neutrinos $(3+1$ scheme $)$ and the neutron to proton decay rates as a function of the new mass eigenstate, the active sterile neutrino mixing angle, and the parameter $a$, in order to obtain the primordial abundances of deuterium, helium and lithium. As in previous works, we have found a sensitivity of the abundances to the active sterile neutrino mixing [14-16, 45]. The value for the parameter $a$ remains lower than 0.60 at $1 \sigma$, in agreement with previous calculations [17]. The results suggest that the primordial abundances are more affected by changes in $a$ and in the neutrino mass rather than in the mixing angle between active and sterile neutrino species.

\section{Acknowledgments}

Support for this work was provided by the National Research Council (CONICET) of Argentina, and by the ANPCYT of Argentina. The authors are members of the Scientific Research Career of the CONICET.

\section{References}

[1] D. Larson et al., Seven-year Wilkinson Microwave Anisotropy Probe (WMAP) Observations: Power Spectra and WMAP-derived Parameters, ApJS 192 (2011) 16.

[2] Planck Collaboration, P. A. R. Ade et al., Planck 2013 results. XVI. Cosmological parameters, $A \&$ A 571 (2014) A16. 
[3] O. Richard, G. Michaud, and J. Richer, Implications of WMAP Observations on Li Abundance and Stellar Evolution Models, ApJ 619 (2005) 538.

[4] J. Meléndez et al., Observational evidence for a broken Li Spite plateau and mass-dependent Li depletion, $A$ \& $A \mathbf{5 1 5}$ (2010) L3.

[5] K. Lind et al., Observational signatures of lithium depletion in the metal-poor globular cluster NGC6397 in IAU Symposium, edited by C. Charbonnel, M. Tosi, F. Primas, \& C. Chiappini (2010), vol. 268 of IAU Symposium, pp. 263 - 268.

[6] O. S. Kirsebom and B. Davids, One fewer solution to the cosmological lithium problem, Phys. Rev. C 84 (2011) 058801.

[7] C. Broggini, L. Canton, G. Fiorentini, and F. L. Villante, The cosmological 7Li problem from a nuclear physics perspective, JCAP 06 (2012) 030.

[8] O. Civitarese and M. E. Mosquera, Nuclear structure bounds on resonant energies: a solution of the cosmological ${ }^{7}$ Li problem?, Nucl. Phys. A 898 (2013) 1.

[9] S. J. Landau, M. E. Mosquera and H. Vucetich, Primordial Nucleosynthesis with varying fundamental constants: A semi-analytical approach, ApJ 637 (2006) 38.

[10] S. J. Landau, M. E. Mosquera, C. G. Scóccola and H. Vucetich, Early universe constraints on time variation of fundamental constants, Phys. Rev. D 78 (2008) 083527.

[11] M. E. Mosquera and O. Civitarese, Time variation of the fine structure constant and of the Higgs vacuum expectation value on cosmological time scales, A \& A 526 (2011) A109.

[12] M. E. Mosquera and O. Civitarese, Effect of the variation of the Higgs vacuum expectation value upon the deuterium binding energy and primordial abundances of $D$ and ${ }^{4} \mathrm{He}, \mathrm{A} \& \mathrm{~A} \mathbf{5 2 0}$ (2010) A112.

[13] O. Civitarese, M. A. Moliné and M. E. Mosquera, Cosmological bounds to the variation of the Higgs vacuum expectation value: BBN constraints, Nucl. Phys. A, 846 (2010) 157.

[14] O. Civitarese and M. E. Mosquera, Testing primordial abundances with sterile neutrinos, Phys. Rev. C 77 (2008) 045806.

[15] O. Civitarese and M. E. Mosquera, Study of the Compatibility of LSND and WMAP Data, IJMPE 17 (2008) 351.

[16] M. E. Mosquera and O. Civitarese, Constraints on active-sterile neutrino mixing from primordial abundances, Phys. Rev. C 84 (2011) 065803.

[17] M. E. Mosquera and O. Civitarese, Sterile neutrinos and Big Bang Nucleosynthesis in the 3+1 scheme, IJMPE 23 (2014) 1450014.

[18] K. N. Abazajian et al., Light Sterile Neutrinos: A White Paper, (2012) arxiv:1204.5379.

[19] D. P. Kirilova and M. V. Chizhov, Cosmological nucleosynthesis and active-sterile neutrino oscillations with small mass differences: The nonresonant case, Phys. Rev. D 58 (1998) 073004.

[20] M. A. Acero and J. Lesgourgues, Cosmological constraints on a light nonthermal sterile neutrino, Phys. Rev. D 79 (2009) 045026.

[21] L. Kawano LET'S GO: EARLY UNIVERSE (1988) FERMILAB-PUB-88-034-A.

[22] L. Kawano LET'S GO: EARLY UNIVERSE II (1992) FERMILAB-PUB-92-004-A.

[23] SNO Collaboration, B. Aharmim et al., Combined analysis of all three phases of solar neutrino data from the Sudbury Neutrino Observatory, Phys.Rev. C $8 \mathbf{8}$ (2013) 025501.

[24] K2K Collaboration, M. H. Ahn et al., Measurement of neutrino oscillation by the K2K experiment, Phys. Rev. D $\mathbf{7 4}$ (2006) 072003. 
[25] GNO Collaboration, M. Altmann et al., Complete results for five years of GNO solar neutrino observations, Phys. Lett. B 616 (2005) 174.

[26] M. Apollonio and A. Baldini, Limits on neutrino oscillations from the CHOOZ experiment, Phys. Lett. B 466 (1999) 415.

[27] Daya Bay Collaboration, F. P. An et al., Observation of Electron-Antineutrino Disappearance at Daya Bay, Phys. Rev. Lett. 108 (2012) 171803.

[28] Double Chooz Collaboration, Y. Abe et al., First measurement of thet $a_{13}$ from delayed neutron capture on hydrogen in the Double Chooz experiment, Phys.Lett. B 723 (2013) 66.

[29] S. A. Balashev, A. V. Ivanchik, and D. A. Varshalovich, $H D / H_{2}$ molecular clouds in the early Universe: The problem of primordial deuterium, Astron. Lett. 36 (2010) 761.

[30] M. Pettini et al., Deuterium abundance in the most metal-poor damped Lyman alpha system: converging on Omega $a_{b, 0} h^{2}$, MNRAS 391 (2008) 1499.

[31] R. J. Cooke et al., Precision Measures of the Primordial Abundance of Deuterium, ApJ 781 (2014) 31.

[32] P. Noterdaeme et al. Deuterium at high redshift. Primordial abundance in the $z_{a b s}=2.621$ damped Ly-alpha system towards CTQ 247, A $\&$ A 542 (2012) L33.

[33] M. Pettini and R. Cooke, A new, precise measurement of the primordial abundance of deuterium, MNRAS 425 (2012) 2477.

[34] Y. I. Izotov and T. X. Thuan, The Primordial Abundance of ${ }^{4} H e:$ Evidence for Non-Standard Big Bang Nucleosynthesis, ApJ $\mathbf{7 1 0}$ (2010) L67.

[35] E. Aver, K. A. Olive and E. D. Skillman, An MCMC determination of the primordial helium abundance, JCAP 04 (2012) 004.

[36] M. Peimbert, V. Luridiana and A. Peimbert, Revised Primordial Helium Abundance Based on New Atomic Data, ApJ 666 (2007) 636.

[37] S. Villanova, G. Piotto and R. G. Gratton, The helium content of globular clusters: light element abundance correlations and HB morphology. I. NGC 6752, A $\&$ A 499 (2009) 755.

[38] E. Aver et al., The primordial helium abundance from updated emissivities, JCAP 11 (2013) 17.

[39] Y. I. Izotov, G. Stasińska and N. G. Guseva, Primordial ${ }^{4}$ He abundance: a determination based on the largest sample of $H$ II regions with a methodology tested on model $H$ II regions, $A \& A$ 558 (2013) A57.

[40] P. E. Nissen and W. J. Schuster, Lithium abundances in high- and low-alpha halo stars, Mem. S. A. It. Suppl. 22 (2012) 41.

[41] L. Sbordone et al., The metal-poor end of the Spite plateau. I. Stellar parameters, metallicities, and lithium abundances, $A \&$ A 552 (2010) A26.

[42] K. Lind et al., Signatures of intrinsic Li depletion and Li-Na anti-correlation in the metal-poor globular cluster NGC 6397, A \& A 503 (2009) 545.

[43] L. Monaco et al., Lithium and sodium in the globular cluster $M$ 4. Detection of a Li-rich dwarf star: preservation or pollution?, A $\&$ A 539 (2012) A157.

[44] Particle Data Group, K. Olive et al., Review of Particle Physics, Chin. Phys. C 38 (2014) 090001.

[45] C. T. Kishimoto, G. M. Fuller, and C. J. Smith, Coherent Active-Sterile Neutrino Flavor Transformation in the Early Universe, Phys. Rev. Lett. 97 (2006) 141301. 